\title{
Tony Gheeraert, Le chant de la grâce. Port-Royal et la poésie d'Arnauld d'Andilly à Racine
}

\section{Benedetta Papasogli}

\section{(2) OpenEdition}

1 Journals

\section{Edizione digitale}

URL: https://journals.openedition.org/studifrancesi/37622

DOI: 10.4000/studifrancesi.37622

ISSN: 2421-5856

\section{Editore}

Rosenberg \& Sellier

\section{Edizione cartacea}

Data di pubblicazione: 15 décembre 2004

Paginazione: 604-605

ISSN: 0039-2944

\section{Notizia bibliografica digitale}

Benedetta Papasogli, «Tony Gheeraert, Le chant de la grâce. Port-Royal et la poésie d'Arnauld d'Andilly à Racine», Studi Francesi [Online], 144 (XLVIII | III) | 2004, online dal 30 novembre 2015, consultato il 08 mai 2021. URL: http://journals.openedition.org/studifrancesi/37622 ; DOI: https://doi.org/10.4000/ studifrancesi.37622

Questo documento è stato generato automaticamente il 8 mai 2021.

\section{(c)}

Studi Francesi è distribuita con Licenza Creative Commons Attribuzione - Non commerciale - Non opere derivate 4.0 Internazionale. 


\title{
Tony Gheeraert, Le chant de la grâce. Port-Royal et la poésie d'Arnauld d'Andilly à Racine
}

\author{
Benedetta Papasogli
}

\section{NOTIZIA}

TONY GHEERAERT, Le chant de la grâce. Port-Royal et la poésie d'Arnauld d'Andilly à Racine, Paris, Champion («Lumière classique»), 2003, pp. 622.

1 Secondo un pregiudizio persistente il giansenismo, ben accordato con la svolta razionalistica che nel cuore del Seicento francese ha preparato il rigore e la sobrietà delle forme classiche, avrebbe contribuito a determinare - dopo la lussureggiante stagione della poesia religiosa barocca - quel crepuscolo della lirica che qualcuno ha chiamato «le nadir de la poésie». L'ampio volume di Tony Gheeraert porta sull'argomento una luce del tutto nuova, mettendo in rilievo la piena partecipazione dei poeti di Port-Royal a una curva culturale tipica del XVII secolo, con un décalage di generazioni evidenziato nel sottotitolo: dalla linfa rigogliosa di Arnauld d'Andilly al sublime sobrio dell'ultimo Racine. Ma, ecco la questione: esistono dunque dei poeti di Port-Royal? La grande novità del volume è nella scoperta stessa e direi quasi nell'"invenzione" del proprio oggetto. Sì, i giansenisti cantavano. La valle dei solitari risuonava di armonie, come, nel Télémaque, il deserto d'Egitto dopo che è stato fatto agli abitanti del "locus terribilis" il dono della musica. Al cuore di quella spiritualità austera, nemica della "fiction" come d'una menzogna, chiusa - fino nei metodi di meditazione - al fascino del sensibile, vi è stato spazio per il gusto e la grazia della poesia.

2 Se il volume che presentiamo si costruisce, imponente, intorno ad un "corpus" tutto sommato assai circoscritto - l'opera poetica di Arnauld d'Andilly, quella di Lemaistre de Sacy, il "Recueil de poésies chrétiennes et diverses" messe sotto l'egida di un poeta 
mondano come La Fontane, la poesia spirituale di Racine, e gli echi del canto giansenista in alcuni amici di Port-Royal - è perché le questioni implicate dalla ricerca sono davvero articolate e profonde. Formuliamo meno ingenuamente la nostra prima domanda: esiste un "canto della grazia" tipico di Port-Royal, quasi una scuola giansenista di lirismo religioso? Per rispondere, l'autore interroga tanto i testi poetici quando le préfaces e gli scritti teorici lasciati dai solitari, la cui cerchia si allarga, di anello in anello, fino a comprendere, non solo l'uno e l'altro Arnauld, Nicole, Pascal, ma ancora Bernard Lamy. Man mano che dall'indagine emergono un'estetica e una morale strettamente intrecciate, lo studioso ha cura di situarle in un vasto orizzonte di idee: nel solco dell'eredità platonica e agostiniana, nel contro luce con l'estetica e con la retorica dei gesuiti, nella dialettica con il cartesianesimo, naturalmente senza ignorare la "querelle" del teatro che fa così spesso dimenticare, col buio delle sue condanne, il contributo degli autori giansenisti alla riflessione sulla poesia.

3 Tony Gheeraert afferma a più riprese il suo debito nei confronti di visioni critiche che gli hanno offerto un fondamentale quadro metodologico: da un lato, la nozione di "Episteme" elaborata da Michel Foucault in Les Mots et les choses, presa qui con cautela come uno strumento operativo per verificare la progressiva trasparenza del segno nella poesia post-barocca; gli studi di Fumaroli sulle retoriche secentesche e sul rapporto fra linguaggio religioso ed immagine; ma anche l'opera capitale di Barbara Lewalski sulla poetica del protestantesimo, messa utilmente a contrasto con i saggi di Philippe Sellier sull'immaginario classico per motivare una scelta critica svolta in una parte centrale del presente volume: il riconoscimento, nella costellazione d'immagini familiari ai poeti giansenisti, non solo la traccia scontata di un immaginario d'epoca, ma il segno di una "imagination de culture" fortemente impregnata da una comune memoria. È un argomento forte per dar corpo all'ipotesi che potrebbe, a momenti, rischiare di far nascere nuovi fantasmi, ossia l'esistenza stessa di una poesia giansenista. Ipotesi, notiamolo, trattata dall'autore fino alla fine con grande prudenza e con un senso particolarmente acuto della complessità culturale, procedendo come attraverso dei "renversements du pour au contre" di cui cercheremo qui di riassumere il movimento.

In un bellissimo capitolo sui rapporti fra i poeti di Port-Royal e la Bibbia, Gheeraert rilegge originalmente la categoria del Dio nascosto come Dio poeta, che si rivela nella natura, nella storia e nella Parola attraverso un regime di figure e di segni, praticando quella retorica divina che nasce da una pietosa "accomodatio" ai bisogni degli uomini: così che l'esegesi biblica diviene per chi la pratica la prima e più alta scuola di linguaggio poetico. Ma subito dopo, ecco che l'intuizione del nesso poesia/profezia, tipica di Port-Royal, è ridimensionata nella sudditanza della poesia, non solo a valori teologici e morali, ma anche a una utilità didattica e persino polemica (con punte verso il burlesco nell'urgenza di ridicolizzare gli avversari gesuiti). E proprio in questo capitolo dedicato alla poesia "servante de la vérité" l'autore ha modo di ridimensionare la specificità delle tematiche gianseniste, alla luce dell'impegno delle prime generazioni di Port-Royal nella vasta campagna teologico-morale della Controriforma. Ma per scommettere subito dopo, in un appassionante capitolo sulle "images obsédantes" dei poeti di Port-Royal, sulla coerenza di un simbolismo che non si diluisce nelle grandi acque di un comune patrimonio secentesco... La forza argomentativa di questo passaggio risulta tuttavia attenuata dagli sviluppi successivi, in cui l'autore pratica un approccio diacronico, e apre fra l'uno e l'altro dei poeti di Port-Royal la frattura stessa che separa - pur attraverso molteplici, insensibili soglie - barocco e classicismo. Arnauld d'Andilly, con la sua ricchezza verbale e con l"'enthousiasme" della sua 
ispirazione, non è forse più vicino al metafisico e visionario Claude Hopil (stranamente qui mai citato) che non ai cantici spirituali di Racine? o forse gli uni e gli altri si ritrovano profondamente in una poetica della rappresentazione, che risucchia e annulla il sensibile, e costituisce il tratto caratterizzate della grande "koiné" poetica del XVII secolo?

5 Non avremo, alla lettura di questo libro, l'emozione di riscoprire valori poetici ignoti o dimenticati. Tony Gheeraert sembra lasciare in bianco uno spazio ultimo del giudizio critico, attribuirsi un compito di approfondimento storico-culturale piuttosto che di critica del gusto e del valore poetico. C'è una prospettiva, ed è quella qui adottata, in cui conta di più afferrare il trascolorare delle stagioni, gli echi e le variazioni dei linguaggi, che non isolare i momenti della purezza e le lunghe note che toccano ancora. Ma avremo il piacere intellettuale di riscoprire nella sua complessità un capitolo mal noto $\mathrm{e}$ spesso addirittura frainteso della letteratura secentesca. Avremo, al termine di un percorso che avrà toccato questioni cruciali della poetica e dell'estetica classica, nei suoi intrecci con la morale e con la teologia, nuove ragioni per stimare la centralità di Port-Royal nella cultura del grande secolo. 